\title{
Condition-dependence, genotype-by-environment interactions and the lek paradox
}

\author{
Hanna Kokko $\cdot$ Katja Heubel
}

Received: 6 February 2007/ Accepted: 13 June 2007/Published online: 7 July 2007

(C) Springer Science+Business Media B.V. 2007

\begin{abstract}
The lek paradox states that maintaining genetic variation necessary for 'indirect benefit' models of female choice is difficult, and two interrelated solutions have been proposed. 'Genic capture' assumes condition-dependence of sexual traits, while genotype-by-environment interactions (GEIs) offer an additional way to maintain diversity. However, condition-dependence, particularly with GEIs, implies that environmental variation can blur the relationship between male displays and offspring fitness. These issues have been treated separately in the past. Here we combine them in a population genetic model, and show that predictions change not only in magnitude but also in direction when the timing of dispersal between environments relative to the life cycle is changed. GEIs can dramatically improve the evolution of costly female preferences, but also hamper it if much dispersal occurs between the life history stage where condition is determined and mating. This situation also arises if selection or mutation rates are too high. In general, our results highlight that when evaluating any mechanism promoted as a potential resolution of the lek paradox, it is not sufficient to focus on its effects on genetic variation. It also has to be assessed to what extent the proposed mechanism blurs the association between male attractiveness and offspring
\end{abstract}

H. Kokko $(\bowtie) \cdot$ K. Heubel

Laboratory of Ecological and Evolutionary Dynamics,

Department of Biological and Environmental Science,

University of Helsinki, Viikinkaari 1, P.O. Box 65, Helsinki

00014, Finland

e-mail: hanna.kokko@helsinki.fi

H. Kokko

School of Botany and Zoology, Australian National University,

Canberra, ACT 0200, Australia fitness; the net balance of these two effects can be positive or negative, and often strongly context-dependent.

Keywords Lek paradox - Genotype-by-environment interaction $\cdot$ Migration $\cdot$ Condition-dependence $\cdot$ Female choice

\section{Introduction}

After decades of research, it is still debated to what extent females can gain indirect (i.e. genetic) benefits through mate choice (e.g. Byers and Waits 2006; Kokko et al. 2006; Qvarnström et al. 2006). A central problem is that of the lek paradox: if female preferences consistently favour some males over others, the favoured genotypes should spread until genetic variation is eroded (Kirkpatrick and Ryan 1991; Rowe and Houle 1996; Hine et al. 2004). The expected indirect benefit should therefore become small and its effects on female preferences easily overridden by any direct effects of mating behaviour on female lifespan or fecundity (i.e. direct fitness) (Kirkpatrick and Ryan 1991).

Two key concepts have increasingly attracted attention in the last decade. First, male sexual traits often show condition-dependence, which helps to explain variation maintenance because the condition of an individual can be assumed to be affected by very many loci, thus providing a 'large target' for mutations (i.e. the 'genic capture' hypothesis, Rowe and Houle 1996; Tomkins et al. 2004). Second, recent work has highlighted the role of genotypeby-environment $(\mathrm{G} \times \mathrm{E})$ interactions (henceforth abbreviated as GEI) as a determinant of male attractiveness (Rodriguez and Greenfield 2003; Hunt et al. 2004; Danielson-François et al. 2006). GEIs mean that genotype expression is influenced by the environment: for example, 
some genotypes of stalk-eyed flies Cyrtodiopsis dalmanni produce males with long eyespans under all conditions, while others only if they are reared in benign environments (David et al. 2000). The reason why GEIs can be relevant for female choice is that if they are strong enough, so that there is no single genotype that is superior over others in all environments (ecological cross-over), they can significantly help to maintain additive genetic variation (Gillespie and Turelli 1989; Turelli and Barton 2004). This can occur in one of two ways: either spatial variation in selection pressures combines with dispersal, or temporal variation combines with overlapping generations (Gillespie and Turelli 1989; Ellner and Hairston 1994; Danielson-François et al. 2006).

Thus, GEIs and condition-dependence together could help to explain costly female choice for indirect benefits (Jia et al. 2000; Hunt et al. 2004). For example, DanielsonFrançois et al. (2006) interpreted that their evidence of significant GEIs in the lesser waxmoth Achroia grisella could prevent the fixation of one genotype that would outperform others across all environments. However, they also discussed a caveat: if a male's fitness is subject to GEIs, then the male's current performance is an unreliable indicator of offspring fitness as soon as the environment changes. This worry has been phrased in a more general context by Greenfield and Rodriguez (2004): condition-dependent signals imply a strong environmentally determined component of male attractiveness, which necessarily makes the signal less reliable as an indicator of offspring fitness, and particularly so when GEIs are operating. Recent experimental evidence, that manipulated litter size as the rearing environment, found partial support for this idea: bank voles show cross-over dominance for male dominance, so that it is no longer heritable from father to son if the rearing environment deteriorated in between, although the effect is attributed to the effects of testosterone rather than condition-dependence per se (Mills et al. 2007).

Thus, GEIs can both help to maintain variation in traits of interest to females, and make the signals unreliable. Do these effects cancel each other out, or is one stronger than the other? Earlier modelling work that focuses on the effects of GEIs has produced results that appear to counter the verbally expressed signal unreliability argument of Greenfield and Rodriguez (2004). Models agree that female preferences for locally adapted males can spread in a spatially structured population (Day 2000; Proulx 2001; Reinhold 2004; McLain 2005), or under different selection regimes (Hughes et al. 2005). This occurs partly because choice, when it favours local male genotypes, can maintain spatial variation rather than removing it (Proulx 2001). However, here we shall show that the optimistic outlook of earlier models is partially compromised, when we join their mathematical modelling approach with the verbal considerations of Greenfield and
Rodriguez (2004) who argued that dispersal between maturation and mating can compromise signal reliability (see their Table 1). We expand this view by considering various options for the timing of dispersal relative to the life cycle of the organism, a factor not varied in earlier models. Dispersal is a crucial parameter because it determines the type of environment offspring experience, as well as the diversity of males that females are exposed to.

Our model explicitly compares the cross-over GEI scenario to an alternative where there is no GEI, while keeping all other features such as overall selection pressure and mutation rates identical. We perform the comparison by predicting the strength of female preferences in each scenario, quantified as the maximal direct cost that female choice can sustain before it is selected against and replaced by random (more generally, cost-minimizing) mating. In addition to dispersal timing, we present results on the effects of the overall magnitude of dispersal, as well as the strength of selection and mutation rates that are required for GEIs to promote female choice.

\section{Modelling GEIs}

We construct a population genetic model that captures the simplest form of condition-dependence with GEIs: an individual's condition is the result of an interaction between the environment and one condition-determining locus, which has two possible alleles $\mathrm{A}$ and a. We assume that there are two different environments, which we label 1 and 2 , and that condition is determined in the environment encountered after dispersal but before mating (and mating does not necessarily take place in the condition-determining environment; for life cycle details see below). While simplistic, our conceptual model allows us to compare scenarios in which there is no GEI so that one genotype performs best in both environments, to a GEI scenario in which there is ecological cross-over. If GEIs promote female choice as predicted by current theory, female choice should be stronger in the latter scenario.

Also, the 'large target' interpretation of Rowe and Houle (1996) is incorporated in the model in a simplified way. The large target assumption offers a way to maintain variation in fitness. The effect of a large number of small mutation rates is a large genome-wide mutation rate, and in a one-locus setting this is best approximated by considering a large range of values for mutation rates between $\mathrm{A}$ and $\mathrm{a}$, including very high values. For simplicity, we assume haploid genetics, thus ignoring genetic dominance which could complicate the analysis.

The condition-determining locus (alleles A and a) is expressed in both sexes, and within each environment, we refer to an individual who has the better allele for this 
environment as an 'individual in good condition'. We assume that being in good condition has two advantages: individuals survive better, and if they are males, their condition allows them to develop superior sexual displays that attract more females. Female behaviour, however, is influenced by a preference locus with two possible alleles $\mathrm{B}$ and $\mathrm{b}$, and they pay attention to male displays only if they express the B allele. These females only mate with males who are in good condition, while females with the $b$ allele mate randomly according to the available proportions of $\mathrm{A}$ and a males. Males carry the preference gene too, but do not express it.

We additionally assume that $B$ females suffer a fecundity cost of magnitude $C$, which allows us to arrive at a measure of the strength of female preferences that can evolve in the system: a threshold value $C^{*}$, above which the preference allele B disappears from the population, is an indication of how large costs females tolerate when choosing males in good condition. Associations between condition-determining and preference-determining loci are not assumed a priori but they can arise through mating biases. The net effect of GEI on female preference evolution can be detected by examining the contrast between scenarios (1) absence of GEI, and (2) model with cross-over GEI. The selection coefficient $s$ is be kept equal between scenarios (1) and (2), so that the only difference between the scenarios is whether the same allele leads to good condition in both environments, or not.

In order for GEIs to exist, individuals born in one environment must occasionally enter the alternative environment. Because one of the main points of our model is to investigate whether the order of life history events matters, we distinguish between dispersal that occurs after the life stage in which condition is determined but before mating, which we for the purpose of illustration assume to occur in males only (thus called male mixing), and dispersal that occurs during any other stage in the life cycle (called migration). For how the terms generalize to other than the spatial scenario envisaged here, see the Discussion.

Assuming discrete generations, the problem can be solved by calculating the evolution of genotype frequencies $\mathbf{p}(t)$ over time. $\mathbf{p}$ is a vector consisting of elements $\left\{p_{\mathrm{ab} 1}\right.$, $\left.p_{\mathrm{aB} 1}, p_{\mathrm{Ab} 1}, p_{\mathrm{AB} 1}, p_{\mathrm{ab} 2}, p_{\mathrm{aB} 2}, p_{\mathrm{Ab} 2}, p_{\mathrm{AB} 2}\right\}$, where the subscripts 1 and 2 refer to the environment in which the frequency is calculated. To obtain more flexible notation, we also use the equivalent indexing $\left\{p_{1}, p_{2}, \ldots, p_{8}\right\} . \mathbf{p}(t)$ refers to frequencies present in newborn offspring. The life cycle consists of several discrete stages, and because each of them alters genotype frequencies, we use notation $\mathbf{p}^{\prime}(t)$, $\mathbf{p}^{\prime \prime}(t)$, etc. when referring to intermediate steps in the life cycle that follow each other until the new generation arises with frequency distribution $\mathbf{p}(t+1)$. The intermediates should be interpreted as densities rather than frequencies because for it is unnecessary to normalize at every stage (this is only necessary when converting results back to newborn gene frequencies, step 7 below).

The life cycle consists of the following steps:

1. Migration: at the beginning of the life cycle, the population consists of newborn offspring $\mathbf{p}(t)$ who initially share the environment of their mother, but can immediately after birth migrate to a new environment. A proportion $m_{1}$ of offspring in environment 1 migrate to environment 2 , and a proportion $m_{2}$ of offspring in environment 2 migrate to environment 1 . Offspring numbers are correspondingly updated to yield $\mathbf{p}^{\prime}(t)$ : e.g. $p_{1}{ }^{\prime}(t)=\left(1-m_{1}\right) p_{1}(t)+m_{2} p_{2}(t)$. Note that migration can, but does not have to, refer to physical movement: 'migration' simply assigns some offspring to rearing conditions that differ in some aspect from others, and this assignment is random if $m_{1}=m_{2}=0.5$. Note also that $\mathbf{p}^{\prime}(t)$ should be interpreted as offspring densities and not frequencies, as they do not necessarily sum up to 1 at this point.

2. Local density regulation occurs within each environment, such that $p_{i}^{\prime \prime}(t)=p_{i}^{\prime}(t) / \sum_{i=1}^{4} p_{i}^{\prime}(t)$ for $1 \leq i \leq 4$, and $p_{i}^{\prime \prime}(t)=p_{i}^{\prime}(t) / \sum_{i=5}^{8} p_{i}^{\prime}(t)$ for $5 \leq i \leq 8$; these boundaries for $i$ correspond to environments 1 and 2 .

3. Selection: viability selection takes place. Two alternative scenarios are studied:

(1) No GEI: allele A leads to good condition in both environments.

(2) GEI: allele A leads to good condition in environment 1 , while allele a leads to good condition in environment 2 .

In both scenarios the new densities of genotypes are given by $\mathbf{p}^{\prime \prime \prime}(t)$, where a fraction $1-s$ of individuals with the allele that leads to poor condition survive in each environment, and the fractions equal unity for all individuals in good condition. For example, $p_{1}^{\prime \prime \prime}(t)=(1-s) p_{1}^{\prime \prime}(t)$ whether or not GEIs are assumed (because $p_{1}=p_{\mathrm{ab} 1}$, and these individuals have the ' $a$ ' allele which always performs poorly in environment 1$)$, while $p_{5}{ }^{\prime \prime \prime}(t)$ equals either $(1-s)$ $p_{5}{ }^{\prime \prime}(t)$ or $p_{5}{ }^{\prime \prime}(t)$ depending on the absence or presence of GEIs (because $p_{5}=p_{\mathrm{ab} 2}$, and the ' $\mathrm{a}$ ' allele performs well in environment 2 but only under the GEI assumption). Note that this definition means that the GEI we model is always strong, in that it corresponds to the definition of ecological cross-over (Hunt et al. 2004; Greenfield and Rodriguez 2004). Scenario (2) should lead to stronger female preferences than (1), if such GEIs have a positive net influence on preference evolution.

4. Male mixing: after condition has been determined and viability selection has taken place, a fraction $x$ of surviving 
males swap environments (migrate from 1 to 2 , or vice versa). Thus, 'mixing' refers to a process that simply determines what kind of males females encounter, without altering the condition of individual males. If $x=0.5$, mixing is complete and females encounter males from each rearing environment equally often, while if $x=0$, females only encounter males with whom they share the environment in which they developed. The updated male distribution is denoted $\mathbf{p}_{\mathrm{m}}{ }^{\prime \prime \prime}(t)$.

5. Mating: mating takes place. Relative proportions of offspring of different types produced in generation $t, \mathbf{q}(t)$, are calculated following the mating preferences of females (b or B) and the rules of haploid inheritance:

$q_{k}=\sum_{i} \sum_{j} p_{i}^{\prime \prime \prime} a_{i j} p_{\mathrm{m} j}^{\prime \prime \prime}\left(1-C_{i}\right) P(i, j \rightarrow k)$.

Here, offspring of type $k$ (e.g. ab1) are produced when females of type $i$ (relative proportion $p_{i}{ }^{\prime \prime \prime}$ ) meet males (relative proportion $p_{\mathrm{m} j}{ }^{\prime \prime \prime}$ ) that are available and acceptable to them $\left(a_{i j}\right)$. For females with the b allele, acceptability $a_{i j}$ has the value 1 if the male (after male mixing) resides in the same environment as the female, and zero otherwise. For $\mathrm{B}$ allele females, the additional requirement for $a_{i j}=1$ is that the male is in good condition. $C_{i}$ is the fecundity cost paid by female of type $i$, and it equals $C$ for females with the $\mathrm{B}$ allele, and 0 otherwise. $P(i, j \rightarrow k)$ denotes the rules of haploid inheritance (excluding mutations): this list of probabilities that an offspring is of type $k$, when the mother is of type $i$ and the father is of type $j$; it is also assumed that the offspring initially resides in the same environment as its mother.

6. Mutation: mutations occur. The mutation rate from A to a is $\mu$, and the back mutation rate is $v$. Post-mutation offspring are described by $\mathbf{q}^{\prime}(t)$. For example, $q_{1}^{\prime}(t)=$ $(1-v) q_{1}(t)+\mu q_{3}(t)$ because mutation in this case flips offspring between states 1 and 3 , equivalent to ab1 and Ab1.

7. The new generation is ready after normalizing:

$\mathbf{p}(t+1)=\mathbf{q}^{\prime}(t) / \Sigma \mathbf{q}^{\prime}(t)$.

The expressions for gene frequency change are unwieldy. To examine whether the allele $B$ persists and spreads with a given cost of preference $C$, the model was analysed numerically. The population was started with otherwise equal frequencies for each genotype, but a low frequency $(0.1)$ for any genotype with preference allele B. The allele B was recorded as not persisting if after 1,000 generations either of the following two conditions was met: the frequency of allele B (averaged over both environments) fell below its initial frequency, or the frequency was decreasing. In practice, these criteria corresponded to fixation of the $b$ allele. We then sought iteratively the threshold value of $C^{*}$, below which allele $\mathrm{B}$ persists according to the criteria above, and above which it is selected against and disappears.

\section{Results}

Whether GEIs improve prospects for female preference evolution depends strongly on the mutation rate (Fig. 1). If mutations are rare, female preferences for indirect benefits disappear without GEIs, but the model with GEIs can maintain additive genetic variation and sustain costly female preferences (Fig. 1). If mutations are very common, however, the absence of GEI can be more favourable for female preferences (Fig. 1a): additive genetic variation is then amply present in any case, and GEIs only decrease signal accuracy as envisaged by Greenfield and Rodriguez (2004). The exact balance depends on parameter values such as migration rates and the strength of selection, and in some cases, GEI appears fairly neutral for preference evolution (peak of Fig. 1b at high mutation rates).

The highest mutation rates considered in Fig. 1 can be considered unrealistic, as condition when modelled as one locus with a high mutation rate is not always equivalent to

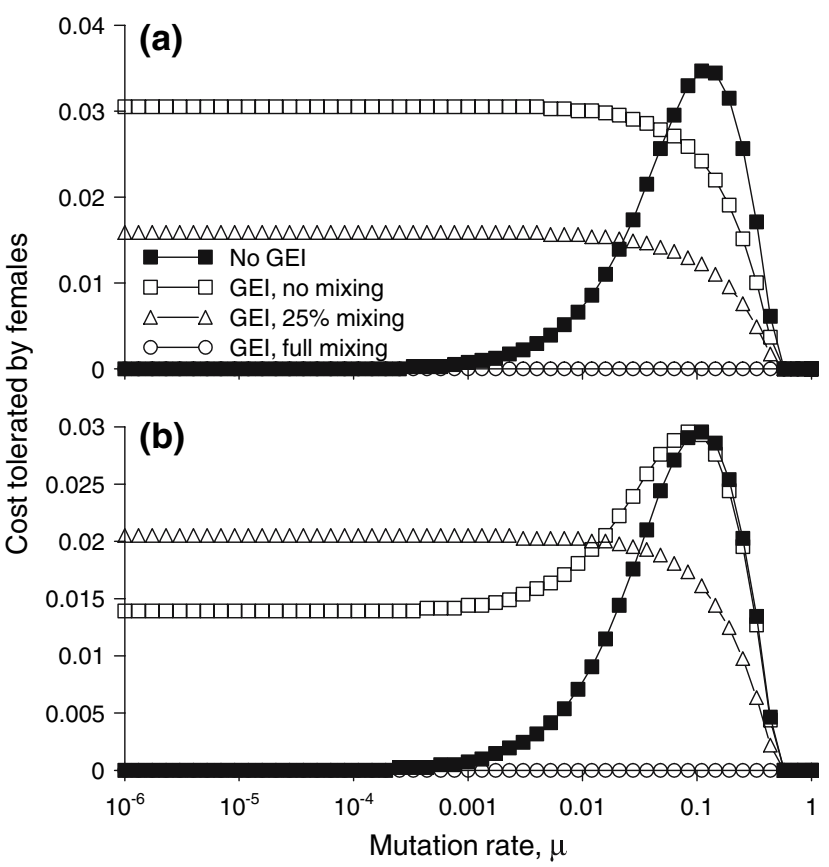

Fig. 1 The strength of female preferences, indicated as the cost $C^{*}$ that preferences tolerate before they disappear from the population, in different circumstances: no GEI (model (1), filled squares), and crossover GEI with $x=0$ (no mixing, open squares), $x=0.25$ (triangles), $x=0.5$ (open dots). Other parameters: (a) $s=0.1, m_{1}=m_{2}=0.2$ and $\mu=v$ as indicated on the axis, (b) likewise, but with selection $s=0.05$, and a much lower migration rate, $m_{1}=m_{2}=0.02$ 
multilocus scenarios with a lower per locus mutation rate (Spichtig and Kawecki 2004). Thus, while we have included these cases for the sake of completeness, it is unclear if mutation rates can be high enough so that GEIs have a negative influence on female choice based on this mechanism alone. However, there is another way in which the ability of GEIs to maintain costly female preferences is compromised, which is considerably more robust as it occurs over all mutation rates: a positive effect of GEIs on preference evolution depends strongly on the assumption that females mainly encounter males within the same environment. If all males mix freely such that females can encounter males from many different rearing environments, female preferences will not evolve under GEIs at all (Fig. 1, open circles). Some mixing is beneficial, however, if migration rates between environments are otherwise low: in Fig. $1 \mathrm{~b}$ at low mutation rates, $25 \%$ male mixing creates stronger female preferences than the absence of male mixing.

This result generalizes in Fig. 2a, where the mixing of males from different environments is systematically varied. Again, high levels of mixing are generally detrimental to female preferences, and near complete mixing ( $x$ close to 0.5 , Fig. 2a) implies that female preferences are stronger without than with GEIs. At low migration and low mixing rates, however, the response can be non-linear: some mixing is favourable and leads to stronger female preferences than no mixing (open triangles in Fig. 2a). Similar results apply to migration in the absence of mixing (Fig. 2b): up to $40 \%$ migration is beneficial and enhances female preference evolution under GEIs, low to moderate migration rates offering the best chances to observe costly female preferences.

Where the parameters (mutation, migration and mixing rates) allow GEIs to enhance female preference evolution, the preference-enhancing effect was found to be quite robust regarding the strength of selection (Fig. 2c). With no selection ( $s=0$, Fig. 2c), solutions with and without GEIs coincide. Some female choice is maintained under this scenario too, and in this case it is purely based on 'sexy son' benefits which can be maintained on their own if the male trait is subject to a negative mutational bias (Pomiankowski et al. 1991; Kokko et al. 2002, 2006). Increasing viability selection, in this case, decreases the frequency of undesirable mates, thereby diminishing the fitness difference of randomly or selectively choosing females. Female preferences in the absence of GEIs therefore evolve less easily when selection against unviable males is strong, but GEIs can strongly counteract this trend (Fig. 2c), by providing a continuous influx of locally maladapted males.

All our results were repeated using a wide range of additional numerical values, including asymmetries in mutation or migration. The results remained similar to the symmetric cases depicted here. However, in cases of strong
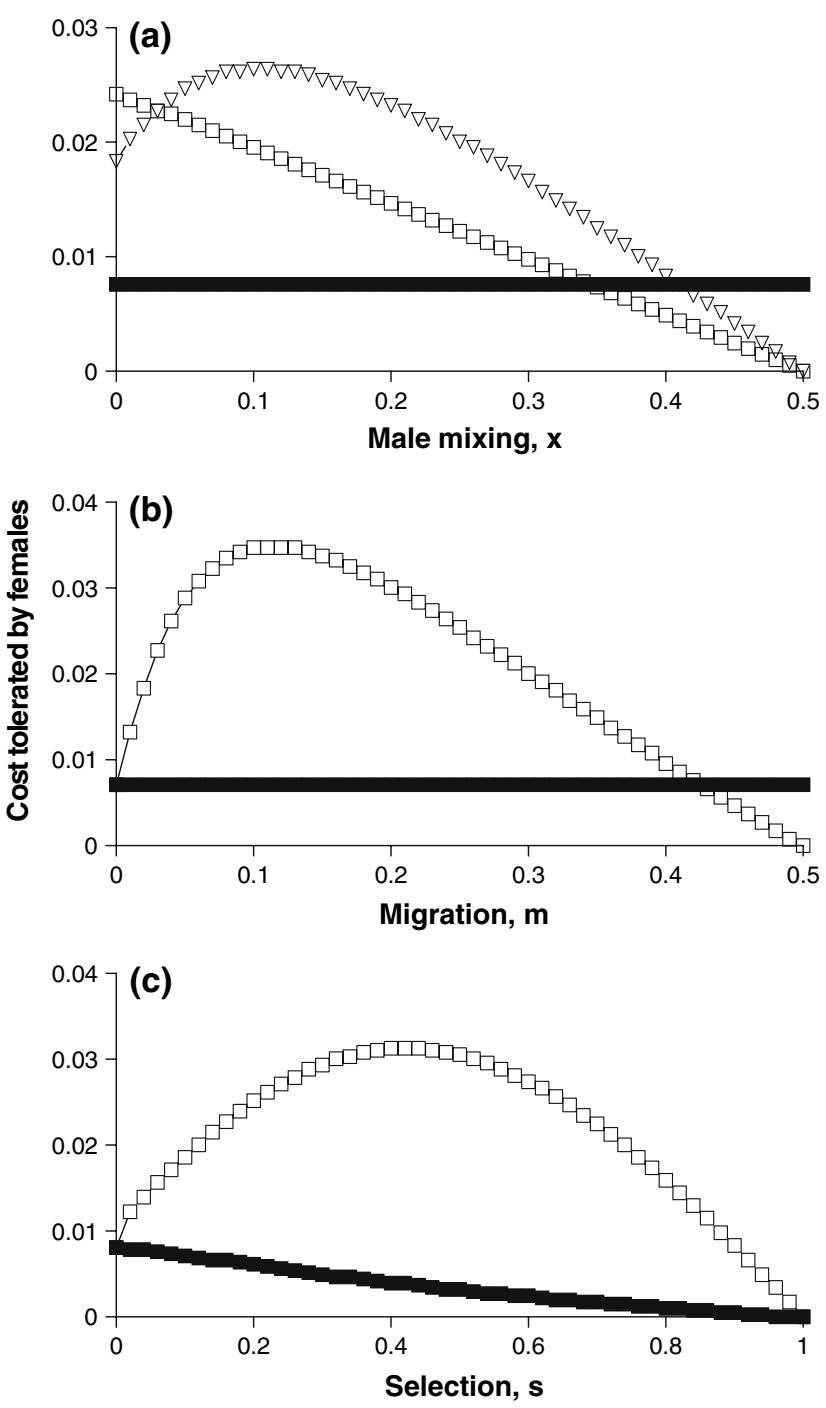

Fig. 2 The strength of female preferences, indicated as in Fig. 1, and plotted against three parameters of the model: (a) male mixing $x$, (b) migration $m_{1}=m_{2}=m$ and (c) selection $s$. Absence of GEIs indicated by black squares, presence of cross-over GEI by open symbols. Parameter values used: (a) $\mu=v=0.01, m_{1}=m_{2}=0.2$ (squares) or 0.02 (triangles), $s=0.05$; different values of $m$ make no difference to the case of no GEIs. (b) $x=0, \mu=v=0.01, s=0.1$; (c) $x=0.2, \mu=v=0.01, m_{1}=m_{2}=0.2$. Using much lower mutation rates yields qualitatively similar results, but with considerably lower black squares, such that GEIs becomes relatively more beneficial for female choice (not shown)

asymmetries, the difference between scenarios with and without GEI became even more prominent: the cost tolerated by females was generally lowered and female choice vanished in cases without GEI. We also introduced asymmetric selection pressures that differed between environments. With selection $s_{1}$ against the poor condition allele in environment and $s_{2}$ in environment 2, scenario (1) no longer corresponds to a total absence of a GEI, but to a weaker form of GEI without crossing reaction norms (see Fig. 1b in Hunt et al. 2004). We found that similar con- 
ditions that made cross-over GEI facilitate female preference evolution when comparing to no GEI (e.g. no excessive male mixing), facilitated preferences in this type of comparison too. Thus, GEIs do not need to be carefully balanced and symmetrical between environments, to have their diversity-promoting influence on local genetic variability; nor do our results depend on the assumption that a particular mutation has advantageous effects in one environment while being deleterious in the other.

\section{Discussion}

A central result from our model is that the relative timing of dispersal compared to the condition-forming time in the life history of the organism is crucially important when using spatial variation to explain the maintenance of female choice. Earlier work (Day 2000; Proulx 2001; Reinhold 2004; McLain 2005) has concluded that GEIs offer a potentially very powerful mechanism that promotes female preference evolution by maintaining variation in male condition, which is then translated to male attractiveness via condition-dependence (see also Zhang 2006). Our results are in line with this work, showing that suitable dispersal rates allow GEIs to strongly boost costly female choice. This occurred despite the fact that we did not assume assortative dispersal, which Greenfield and Rodriguez (2004) suspected could be necessary to maintain signal reliability and hence female choice. This positive result occurs when females mostly mate with males who are fit in the environment in which both the male and the female currently reside, and when dispersal, selection and mutation rates are suitable (very high or low extremes make GEIs detrimental to female choice).

However, our conclusion that GEIs promote preference evolution, changes considerably when we make a particular assumption regarding timing of dispersal: that some males move between environments after their condition has been determined, but before matings occur. The effect of such 'male mixing' is dramatically non-linear. Some degree of mixing can enhance the positive effects of GEIs on choice evolution, but if males in the mating pool have too diverse origins, much less female choice can typically be maintained in the presence of GEIs than if GEIs are absent. GEIs then mean that females encounter males that have encountered different environments during the life history stages that are crucial for developing his condition.

Why is some mixing beneficial while too much of it is harmful? Any process that maintains variation in male traits has some potential to explain costly female preferences, because preferences are obviously pointless unless potential mates differ from each other. But this does not mean that more variation always favours more choice: variation-generating mechanisms can also blur the association between 'what the female sees and what she gets'. As a thought experiment, imagine, e.g. enormous mutation rates between $\mathrm{A}$ and $\mathrm{a}$ : this would obviously maintain much variation, but in the extreme $(\mu=v=0.5$ in our model) this simultaneously means that parental genotypes no longer predict what alleles offspring inherit, which again makes mate choice pointless. It is important to realize that the positive and negative effects of variationmaintaining mechanisms generally do not cancel out, instead the net balance can remain either positive or negative (Kokko et al. 2007). In the case of male mixing, this balance depends on how much mixing there is. Some mixing gives reason for females to evolve preferences to avoid maladapted immigrant males as mates, but a too diverse mixture of males with different backgrounds is bad news for female preference evolution: some males will appear attractive simply because their condition was improved by environments that will not be equivalent to the current ones, or those that the female's own offspring will encounter.

Many factors considered in our model have a similarly non-linear effect, which repeatedly highlights that the balance between positive and negative effects does not need to coincide under spatial variation and can depend on the relative strength of each process. For example, too small selection against poorly performing genotypes (small $s$ ) implies that females do not gain much by being choosy, whereas too strong selection (large $s$ ) implies that badly performing males are wiped out by viability selection, thus there is again little reason to choose. Intermediate values of $s$ thus create the best prospects for costly choice. Similarly with migration: too little does not maintain the presence of different types of individuals within a patch, too much of it simply swamps a population to be uniform again.

Greenfield and Rodriguez (2004) pointed out that it becomes puzzling how female choice based on indirect male benefits can evolve when GEIs blur the relationship between the male signal and offspring performance. Qvarnström (1999) similarly suggests that indirect benefits of mate choice are reduced in fluctuating or heterogeneous environments (see also Collins et al. 1999). On the other hand, Mills et al. (2007) suggest that variable environments (density cycles in the context of their study species, the bank vole) can instead help by maintaining alternative genotypes, while Kokko et al. (2007) consider a special case-preference for rare male phenotypes-and show how imperfectly expressed female choice can aid preference evolution rather than hinder it because it helps to maintain more variation. Our results reconcile these diverse views, by showing that it is not sufficient to focus on one side of the coin only, i.e. the examination of how much more additive genetic variation can be maintained if GEIs 
operate. The other side of the coin is to assess how strongly GEIs blur the relationship between male display and offspring performance, among characteristic samples of males that are available to females under natural conditions. It is important to note that GEI studies conducted in the laboratory are prone to miss this aspect.

This highlights the need for more integrative work, since the balance of the two sides determines whether GEIs have a positive or negative net effect on female choice. This balance can be influenced by factors such as spatial versus temporal variation and their predictability, whether dispersal is random with respect to adaptedness of an individual to its locality (as an alternative, individuals may settle once they encounter environments that suit them), and whether the condition and hence attractiveness of males is determined by recent or older environmental conditions: longer time lags probably make the signals less reliable. One can expect these diverse factors to flip the sign of the net effect on choice evolution easily from positive to negative, and hence our model should be considered a first step in quantifying the direction of the expected net effects. Condition-dependence of female preferences (Cotton et al. 2006) or various forms of ontogenetic conflict where traits favoured in males may be detrimental for females (Mills et al. 2007) could potentially enhance the diversity of potential outcomes further.

In our model, the relationship between display and offspring fitness is blurred, making GEIs detrimental for female choice, if males move between environments after their condition is determined (but before mating). However, as emphasized by Greenfield and Rodriguez (2004), the detrimental effect probably generalizes to other scenarios. The negative effects of 'male mixing' are based on the fact that it makes a male's ornament (or current condition) a relatively unreliable signal of his adaptation to the current environment. Thus, any process that blurs the relationship between the signal and offspring fitness should have a similar effect. Examples include extensive female mate sampling that reaches males that developed in different environments (McLain 2005), and environmental determination of sexually selected traits (Griffith et al. 1999), which can also combine with cohort effects (Ellner and Hairston 1994; Lindström and Kokko 2002) in species in which generations overlap and conditions early in life are a major determinant of condition (Lindström 1999).

The last example is a particularly relevant one because GEIs have also been shown to enhance the maintenance of additive genetic variation when there is temporal variation in selection and generations overlap (Ellner and Hairston 1994), while our model considers GEIs in the alternative setting with spatial variation in selective pressures and dispersal between environments (also interpret as microenvi- ronments, as the distances dispersed could be physically short, e.g. different host plants growing in a mixed forest). While our model with its one condition-determining locus is a definite oversimplification of natural systems, the diversity of outcomes that even such a simple model shows suggests that any model or empirical dataset concerned with GEIs and female choice should assess how the range of males that females encounter influences the results.

To our knowledge, there is no data to date to test our predictions that female preference evolution should be hampered rather than aided by GEIs in species in which condition is largely determined by conditions before dispersal, or in long-lived species with strong cohort effects, or in species in which mutation rates are so high that mutation-selection balance would by itself, without any spatial or temporal environmental variation, be able to maintain strong female preferences. Because the effect of a GEI on female preference evolution can completely change from strongly positive to strongly negative, population or species comparisons on this issue would be extremely welcome. In general, our results stress how evaluating any candidate mechanism's power as a potential resolution of the lek paradox should not focus solely on the effects on genetic variation. Indeed, it is difficult to envisage variance-maintaining mechanisms that would not simultaneously make a choosy female less certain about 'what she sees is what she gets', in other words, weaken the correlation between preference (or male attractiveness) and offspring fitness. But as shown here, these two effects do not necessarily cancel out, thus quantitative predictions will be needed in each case.

Acknowledgements We thank the editors of this issue for inviting us to write this paper, two anonymous reviewers for their constructive suggestions, Michael Jennions for comments and coffee, Michael Greenfield and Jan Lindström for fruitful discussions, and the Academy of Finland for funding.

\section{References}

Byers JA, Waits L (2006) Good genes sexual selection in nature. Proc Natl Acad Sci 103:16343-16345

Collins RD, Jang Y, Reinhold K, Greenfield MD (1999) Quantitative genetics of ultrasonic advertisement signalling in the lesser waxmoth Achroia grisella (Lepidoptera: Pyralidae). Heredity 83:644-651

Cotton S, Small J, Pomiankowski A (2006) Sexual selection and condition-dependent mate preferences. Curr Biol 16:R755-R765

Danielson-François AM, Kelly JK, Greenfield MD (2006) Genotype x environment interaction for male attractiveness in an acoustic moth: evidence for plasticity and canalization. J Evol Biol 19:532-542

David P, Björksten T, Fowler K, Pomiankowski A (2000) Conditiondependent signalling of genetic variation in stalk-eyes flies. Nature 406:186-188 
Day T (2000) Sexual selection and the evolution of costly female preferences: spatial effects. Evolution 54:715-730

Ellner S, Hairston NG (1994) Role of overlapping generations in maintaining genetic variation in a fluctuating environment. Am Nat 143:403-417

Gillespie JH, Turelli M (1989) Genotype-environment interactions and the maintenance of polygenic variation. Genetics 10:253280

Greenfield MD, Rodriguez RL (2004) Genotype-environment interaction and the reliability of mating signals. Anim Behav 68:1461-1468

Griffith SC, Owens IPF, Burke T (1999) Environmental determination of a sexually selected trait. Nature 400:358-360

Hine E, Chenoweth SF, Blows MW (2004) Multivariate quantitative genetics and the lek paradox: genetic variance in male sexually selected traits of Drosophila serrata under field conditions. Evolution 58:2754-2762

Hughes KA, Rodd FH, Reznick DN (2005) Genetic and environmental effects on secondary sex traits in guppies (Poecilia reticulata). J Evol Biol 18:35-45

Hunt J, Bussiere LF, Jennions MD, Brooks R (2004) What is genetic quality? Trends Ecol Evol 19:329-333

Jia FY, Greenfield MD, Collins RD (2000) Genetic variance of sexually selected traits in waxmoths: maintenance by genotype $\mathrm{x}$ environment interaction. Evolution 54:953-967

Kirkpatrick M, Ryan MJ (1991) The evolution of mating preferences and the paradox of the lek. Nature 350:33-38

Kokko H, Brooks R, McNamara JM, Houston AI (2002) The sexual selection continuum. Proc R Soc Lond B 269:1331-1340

Kokko H, Jennions MD, Brooks R (2006) Unifying and testing models of sexual selection. Ann Rev Ecol Evol Syst 37:4366

Kokko H, Jennions MD, Houde A (2007) Evolution of frequencydependent mate choice: keeping up with fashion trends. Proc R Soc Lond B 274:1317-1324

Lindström J (1999) Early development and fitness in birds and mammals. Trends Ecol Evol 14:343-348

Lindström J, Kokko H (2002) Cohort effects and population dynamics. Ecol Lett 5:338-344
McLain DK (2005) Female soldier beetles display a flexible preference for selectively favored male phenotypes. Evolution 59:1085-1095

Mills SC, Alatalo RV, Koskela E, Mappes J, Mappes T, Oksanen TA (2007) Signal reliability compromised by genotype by environment interaction is preserved by ontogenetic conflict. Evolution 61:1748-1757

Pomiankowski A, Iwasa Y, Nee S (1991) The evolution of costly mate preferences. 1. Fisher and biased mutation. Evolution 45:1422-1430

Proulx SR (2001) Female choice via indicator traits easily evolves in the face of recombination and migration. Evolution 55:24012411

Qvarnström A (1999) Genotype-by-environment interactions in the determination of the size of a secondary sexual character in the collared flycatcher (Ficedula albicollis). Evolution 53:15641572

Qvarnström A, Brommer JE, Gustafsson L (2006) Testing the genetics underlying the co-evolution of mate choice and ornament in the wild. Nature 441:84-86

Reinhold K (2004) Modeling a version of the good-genes hypothesis: female choice of locally adapted males. Org Divers Evol 4:157163

Rodriguez RL, Greenfield MD (2003) Genetic variance and phenotypic plasticity in a component of female mate choice in an ultrasonic moth. Evolution 57:1304-1313

Rowe L, Houle D (1996) The lek paradox and the capture of genetic variance by condition dependent traits. Proc R Soc Lond B 263:1415-1421

Spichtig M, Kawecki TJ (2004) The maintenance (or not) of polygenic variation by soft selection in heterogeneous environments. Am Nat 164:70-84

Tomkins JL, Radwan J, Kotiaho JS, Tregenza T (2004) Genic capture and resolving the lek paradox. Trends Ecol Evol 19:323-328

Turelli M, Barton NH (2004) Polygenic variation maintained by balancing selection: Pleiotropy, sex-dependent allelic effects and GxE interactions. Genetics 166:1053-1079

Zhang X-S (2006) The phenotypic variance within plastic traits under migration-mutation-selection balance. Evolution 60:1125-1136 